\title{
Application of Inverse Gamma Transport to Material Thickness Identification with SGRD Code.
}

\author{
Philippe Humbert ${ }^{*}$ \\ CEA DAM Ile-de-France, Bruyères-le-Châtel, 91297 Arpajon cedex, France
}

\begin{abstract}
SGRD (Spectroscopy, Gamma rays, Rapid, Deterministic) code is used to infer the dimensions of a one dimensional model of a shielded gamma ray source. The method is based on the simulation of the uncollided leakage current of discrete gamma lines that are produced by nuclear decay. Experimentally, the unscattered gamma lines leakage current is obtained by processing high precision gamma spectroscopy measurements. The material thicknesses are computed with SGRD using a fast ray-tracing algorithm embedded in a non-linear multidimensional iterative optimization procedure that minimizes the error metric between calculated and measured signatures. For verification, numerical results on a test problem are presented.
\end{abstract}

\section{Introduction}

Identification of nuclear radiation source characteristics is a subject of interest for nonproliferation and nuclear safeguard applications. Gamma spectroscopy is used for this purpose due to the sensitivity of these measurements to source isotopic composition and shielding materials properties. The determination of source characteristics using known signature measurements is an inverse transport problem. This subject has been studied by different authors (see for example [1-5]).

Here, we present the determination of the unknown material interface positions from the measured uncollided gamma line spectrum obtained by processing high precision gamma spectroscopy measurements. For this purpose, SGRD code [6], which was designed for fast computation of gamma ray leakage out of 1-D spherical assemblies, is used as a forward solver for iterative inverse transport calculations. The material thicknesses are computed using a non-linear multidimensional iterative optimization algorithm that minimizes the error metric between calculated and measured signatures. The optimization is performed using the gradient free Powell method $[8,9]$.

For verification, numerical results on a test problem are presented. A synthetic gamma lines spectrum is used as input to the inverse transport solver and the obtained geometry is compared to the original one.

\section{Uncollided Leakage Gamma Current Calculation}

\subsection{Gamma leakage}

The observable is the uncollided gamma ray leakage line spectrum produced by the radioactive decay gamma source.

The number $L$ of gamma particles leaving a sphere of radius $R$ per unit time and solid angle without collision is calculated using the uncollided angular flux $\psi(R, \mu)$.

$$
L=2 \pi R^{2} \int_{0}^{1} \mu \psi(R, \mu) d \mu
$$

In equation (1), $\mu$ is the cosine of the angle between the particle direction of flight and the spatial radial vector on the external surface.

\subsection{Ray tracing}

The direct flux is solution of the transport equation without scattering. It is solved by SGRD code using an accurate and fast ray-tracing method which gives the exact solution of the integral transport equation along discrete directions.

The shielded source is made of concentric spherical shells, each with a constant source intensity $Q_{i}$ and total macroscopic cross section $\sigma_{i}$. As illustrated in figure 1 , the angular discretization is such that a set of $N_{i}$ characteristic rays is associated to each shell, from the most internal $(i=1)$ to the most external shell with a nonnull source $(i=I)$.

\footnotetext{
* Corresponding author: philippe.humbert@cea.fr
} 


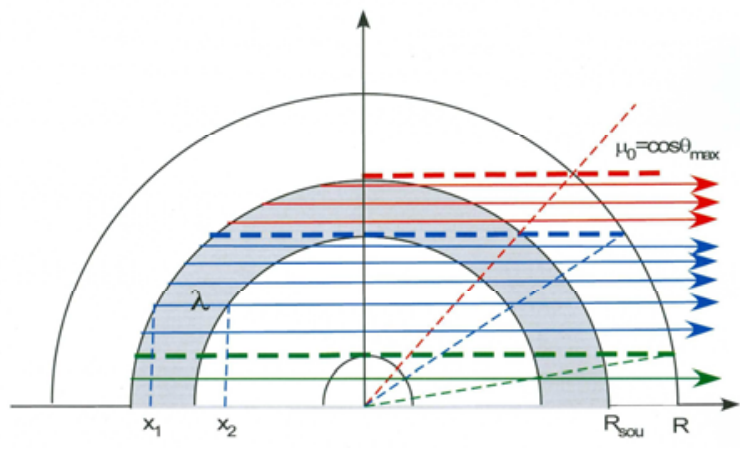

Fig. 1. 1-D spherical ray-tracing. The $\gamma$ source is in the shaded shell.

The unscattered leakage is given by :

$$
L=2 \pi R^{2} \sum_{i=1}^{I} \frac{\Delta \mu_{i}}{N_{i}} \sum_{j=1}^{N_{i}} \mu_{i j} \psi\left(R, \mu_{i j}\right)
$$

In equation (2):

$\mu_{i j}$ characterizes the direction of flight associated to the $j^{\text {th }}$ ray crossing the $i^{\text {th }}$ shell,

$\mu_{i}$ is the cosine associated to the upper boundary of shell $i$ and $\Delta \mu_{\mathrm{i}}=\mu_{i}-\mu_{i-1}$.

The angular flux $\psi$ is calculated using the following transmission equation:

$$
\psi\left(x_{i+1}, \mu_{j}\right)=\psi\left(x_{i}, \mu_{j}\right) e^{-\sigma_{i} \lambda_{i}}+\frac{Q_{i}}{\sigma_{i}}\left(1-e^{-\sigma_{i} \lambda_{i}}\right)
$$

The $\lambda_{\mathrm{i}}$ are the intersection lengths of the characteristics with the spherical shells.

$$
\lambda_{i}=x_{i+1}-x_{i} \text { with } x_{i}=\sqrt{R_{i}^{2}-R^{2}\left(1-\mu^{2}\right)}
$$

\subsection{Validation}

The particle leakage from an homogeneous spherical source of radius $\mathrm{R}$, opacity $\sigma$ and intensity $\mathrm{Q}$ has an analytic expression [7].

$$
L=\frac{Q}{8 \sigma^{3}}\left[(1+2 \sigma R) \exp (-2 \sigma R)+\frac{(2 \sigma R)^{2}}{2}-1\right]
$$

The convergence of the ray tracing algorithm with the number of directions is illustrated in Table 1 for $\mathrm{R}=5 \mathrm{~cm}, \sigma=1.484673 \mathrm{~cm}^{-1}$ and $\mathrm{Q}=1948.531 \mathrm{~cm}^{-3} \cdot \mathrm{s}^{-1}$.

The ray tracing solver is faster and more accurate than $\mathrm{S}_{\mathrm{N}}$ discrete ordinates for unscattered transport calculations (cf. figure 2).
Table 1. Convergence of the ray tracing and $S_{N}$ solvers with the number of directions.

\begin{tabular}{|c|c|c|}
\hline $\begin{array}{c}\text { Number of } \\
\text { directions }\end{array}$ & $\begin{array}{c}\mathrm{S}_{\mathrm{N}} \text { Leakage } \\
(100 \text { cells })\end{array}$ & $\begin{array}{c}\text { Ray-tracing } \\
\text { Leakage }\end{array}$ \\
\hline 8 & 8188.1891 & 8120.3737 \\
\hline 16 & 8142.4987 & 8125.7923 \\
\hline 32 & 8131.6601 & 8127.6136 \\
\hline 64 & 8129.0541 & 8128.1026 \\
\hline 128 & 8128.4142 & 8128.2270 \\
\hline 256 & 8128.2552 & 8128.2583 \\
\hline 512 & 8128.2155 & 8128.2661 \\
\hline 1024 & 8128.2056 & 8128.2680 \\
\hline Analytic & \multicolumn{2}{|c|}{$\mathbf{8 1 2 8 . 2 6 7 9}$} \\
\hline
\end{tabular}

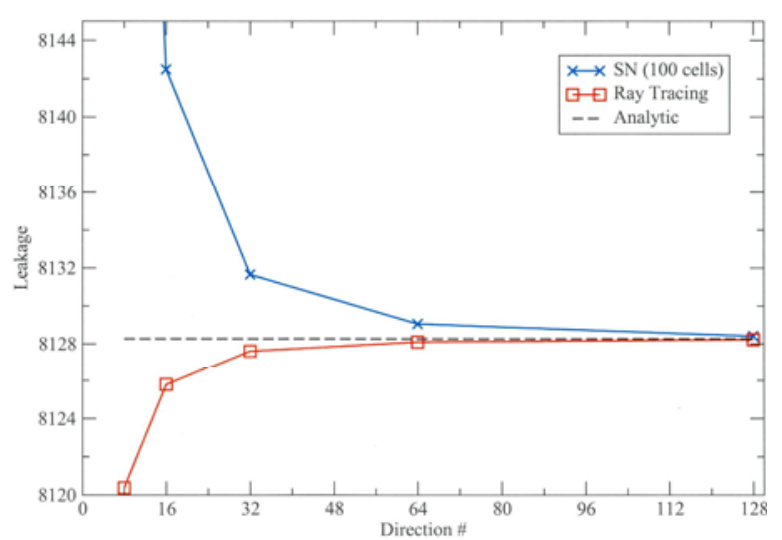

Fig. 2. Convergence to the analytic solution of the ray tracing and $\mathrm{S}_{\mathrm{N}}$ leakage calculations with the number of directions.

\section{Optimization - Inverse Transport}

The goal of the inverse transport problem is to find the 1-D spherical assembly of source and shielding materials that produces the better agreement with the measured uncollided leakage gamma spectrum. Starting from a model composed of a given number of material atomic compositions and densities, the optimization algorithm automatically searches the shell thicknesses that give the best estimate of the measured line spectrum. The goal is then to minimize the error or distance between the measured and calculated spectrum. The quality of the estimation is quantified by the chosen error metric. 


\subsection{Error Metric}

We use a least square method, the error is defined as :

$$
E\left(x_{1}, x_{2}, \ldots, x_{M}\right)=100 \sqrt{\frac{1}{N} \sum_{i=1}^{N} \omega_{i}\left(\frac{L_{i}^{\text {mes }}-L_{i}^{\text {calc }}}{L_{i}^{\text {mes }}}\right)^{2}}
$$

$$
\begin{array}{ll}
L_{i}^{\text {mes }} & : \text { Measured leakage in line } i \\
L_{i}^{\text {calc }} & : \text { Calculated leakage in line } i, \\
M & : \text { Number of material shells, } \\
N & : \text { Number of spectral lines, } \\
x_{m} & : \text { Thickness of shell } m, \\
\omega_{i} & : \text { Weight of line } i .
\end{array}
$$

Geometric or mass constraints can be incorporated in the error function. For instance one can favor the external radius around $R=R_{0}$ using the following error function where $\lambda$ is the weight associated to the constraint.

$E(\%)=100 \sqrt{\frac{1}{N} \sum_{i=1}^{N} \omega_{i}\left(\frac{L_{i}^{\text {mes }}-L_{i}^{\text {calc }}}{L_{i}^{\text {mes }}}\right)^{2}+\lambda\left(\frac{R-R_{0}}{R_{0}}\right)^{2}}$

\subsection{Stochastically Restarted Powell Optimization Method}

The Powell method [8,9] is a multidimensional deterministic optimization algorithm which proceeds by a sequence of line minimizations along well chosen directions.

One advantage of the Powell method is that it needs to evaluate only the error function and does not necessitate the calculation of the gradient of the error with respect to the optimized variables for the choice of the successive minimization directions.

The Powell's method discarding the direction of largest decrease described in [8] is used to avoid the generation of linearly dependent set of directions.

The line minimizations are performed by successive bracketing of the minimum using Brent's method which switches between inverse parabolic interpolation and golden section.

If the error function has local minima, the Powell method can miss the best solution. In order to move from a local minimum the method can be stochastically restarted and another minimum is searched, the global minimum is estimated as the smallest local minimum after a given number of iterations.

We have implemented the following heuristic algorithm. The Powell optimization is embedded within a simple random optimization loop which is periodically restarted (cf. figure 3 ). The external radius of each shell is randomly sampled and the best configuration according to the error metric is retained and serves for a new Powell optimization. The best Powell's optimized geometry is retained.

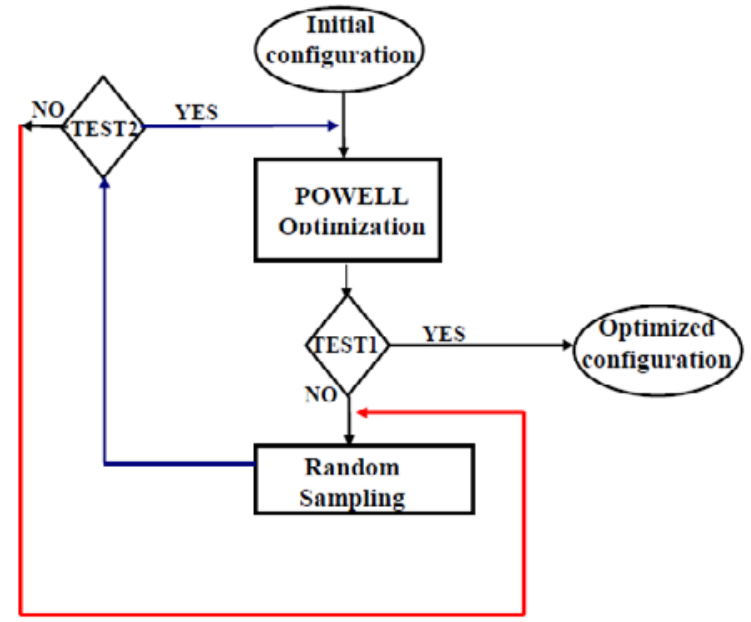

Fig. 3. Heuristic optimization algorithm : Powell optimization embedded within a simple random optimization loop.

In figure 3, TEST1 means that the total number of iterations has reached a maximum (200 for instance). The algorithm ends when TEST1 is true. TEST2 means that the number of random iterations is equal to a maximum ( 10 for instance) or that the random configuration is better than the previous ones. The Powell's optimization is triggered only when TEST2 is true. The loop in figure 3 is used to find a new random restart for the Powell's iterative algorithm which is not detailed in the figure.

\section{SGRD Code}

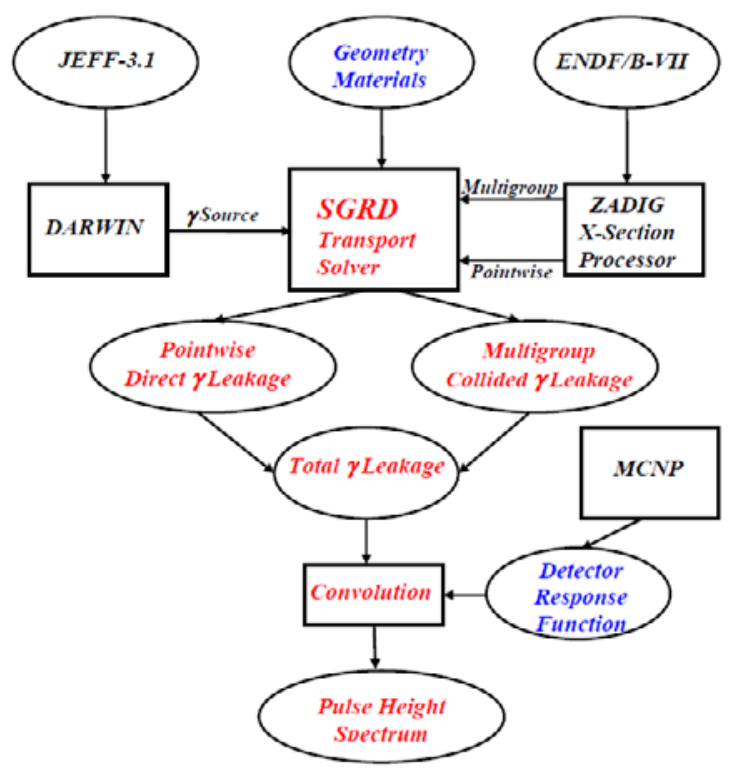

Fig. 4. SGRD input/output flow diagram for gamma spectra calculation.

SGRD code is primarily designed for fast computation of gamma pulse height spectra produced by spherical 
shielded sources [10]. The ray tracing solver is used for direct gamma lines transport and the collided flux is calculated using a discrete ordinates $\mathrm{S}_{\mathrm{N}}$ solver, the gamma spectrum is obtained by convolution with the detector response function (cf. figure 4).

The code has been adapted for material interface position determination using the direct gamma lines intensity extracted from the measured gamma spectrum. For this purpose the Ray tracing solver is embedded in the stochastically restarted Powell minimization algorithm (cf. figure 5).

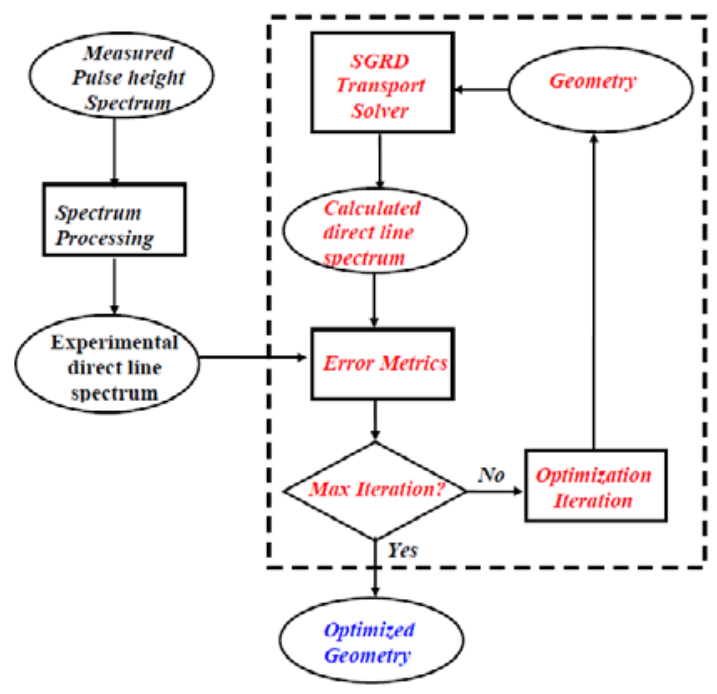

Fig. 5. Inverse transport for geometry search using SGRD ray-tracing solver.

A typical computation time for the optimization process is of the order of seconds or less on a personal computer $\left(\right.$ DELL $^{\circledR}$ precision T5600).

\section{Test Problem - Numerical Results}

The test problem is a spherical natural uranium shell surrounded by polyethylene $\left(\mathrm{CH}_{2}\right)$ and an aluminum cover (cf. table 2).

Table 2. Test problem specification.

\begin{tabular}{|c|l|c|c|c|}
\hline Materials & Air & Uranium & $\mathrm{CH}_{2}$ & Aluminum \\
\hline $\begin{array}{l}\text { Atomic } \\
\text { Comp. }\end{array}$ & $\begin{array}{c}{ }^{16} \mathrm{O} 20 \% \\
{ }^{14} \mathrm{~N} 80 \%\end{array}$ & $\begin{array}{c}{ }^{238} \mathrm{U} 99.3 \% \\
{ }^{235} \mathrm{U} 0.7 \%\end{array}$ & $\begin{array}{c}{ }^{12} \mathrm{C} 1 / 3 \\
\mathrm{H} 2 / 3\end{array}$ & ${ }^{27} \mathrm{Al} 100 \%$ \\
\hline $\begin{array}{c}\text { Density } \\
\left(\mathrm{g} / \mathrm{cm}^{3}\right)\end{array}$ & 0.0013 & 18.9 & 0.9 & 2.7 \\
\hline $\begin{array}{c}\mathrm{R}_{\mathrm{EXT}} \\
(\mathrm{cm})\end{array}$ & 18 & 20 & 29 & 30 \\
\hline
\end{tabular}

The gamma source is due to the uranium radioactive decay. Five characteristic lines are chosen for the optimization (cf. table 3 ).
Table 3. $\gamma$ line characteristics and simulated leakage measurements.

\begin{tabular}{|l|c|l|c|}
\hline Isotope & $\begin{array}{c}\text { Energy } \\
(\mathrm{keV})\end{array}$ & $\begin{array}{c}\text { Intensity } \\
(\gamma / \mathrm{g} / \mathrm{s})\end{array}$ & $\begin{array}{c}\text { Leakage } \\
(\gamma / \mathrm{s} / \mathrm{s})\end{array}$ \\
\hline \multirow{3}{*}{${ }^{235} \mathrm{U}$} & 143.76 & 8753 & 282.86 \\
\cline { 2 - 4 } & 185.71 & 45660.2 & 3259.60 \\
\cline { 2 - 4 } & 205.31 & 4004.4 & 386.95 \\
\hline${ }^{238} \mathrm{U}$ & 766.36 & 39.7 & 12604 \\
\cline { 2 - 4 } & 1001.03 & 103.8 & 50599 \\
\hline
\end{tabular}

The measurements are simulated using SGRD uncollided direct transport calculations.

A first optimized configuration is found after 10 Powell iterations. The external radius of the uranium and polyethylene shells are yet close to the solution, but the internal radius of the uranium shell is still wrong. In this case a correct solution is found after 159 iterations with several stochastic restarts as shown in figure 6 where the error value is plotted versus the number of iterations.

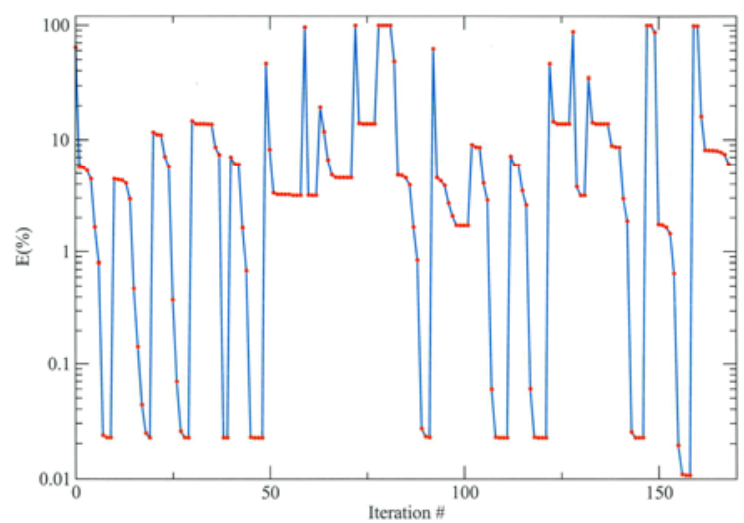

Fig. 6. Evolution of the error metric with the number of iterations.

The results of the optimization process using Powell method are presented in table 4 and 5.

Table 4. Results of the optimization, external radius.

\begin{tabular}{|c|c|c|c|c|}
\hline Materials & Air & Uranium & $\mathrm{CH}_{2}$ & Aluminum \\
\hline Reference $\mathrm{R}_{\mathrm{EXT}}$ & 18 & 20 & 29 & 30 \\
\hline $\begin{array}{c}\text { Initialized } \mathrm{R}_{\mathrm{EXT}} \\
\text { Iteration\# } \\
\mathrm{E}=85.319 \%\end{array}$ & 9 & 16 & 23 & 30 \\
\hline $\begin{array}{c}\text { Optimized } \mathrm{R}_{\mathrm{EXT}} \\
\text { Iteration\# 10 } \\
\mathrm{E}=0.0226 \%\end{array}$ & 13.433 & 19.993 & 29.008 & 30.000 \\
\hline $\begin{array}{c}\text { Optimized } \mathrm{R}_{\mathrm{EXT}} \\
\text { Iteration\# 159 } \\
\mathrm{E}=0.0108 \%\end{array}$ & 17.967 & 19.998 & 29.002 & 30.000 \\
\hline
\end{tabular}


Table 5. Results of the optimization, gamma lines intensities.

\begin{tabular}{|l|l|c|c|}
\hline $\begin{array}{c}\text { Energy } \\
(\mathrm{keV})\end{array}$ & \multicolumn{1}{|c|}{$\begin{array}{c}\text { Initial } \\
(\gamma / \mathrm{sr} / \mathrm{s})\end{array}$} & $\begin{array}{c}\text { Optimized } \\
(\gamma / \mathrm{sr} / \mathrm{s})\end{array}$ & $\begin{array}{c}\text { "Measured' } \\
(\gamma / \mathrm{sr} / \mathrm{s})\end{array}$ \\
\hline 143.76 & 21.30 & 282.89 & 282.86 \\
\hline 185.71 & 309.75 & 3259.80 & 3259.60 \\
\hline 205.31 & 39.78 & 386.88 & 386.95 \\
\hline 766.36 & 2815.44 & 12604.36 & 12604 \\
\hline 1001.03 & 12807.21 & 50601.19 & 50599 \\
\hline
\end{tabular}

The comparison between simulated (direct transport calculation), initial and optimized spectra (inverse transport calculation) is presented in figure 7 .

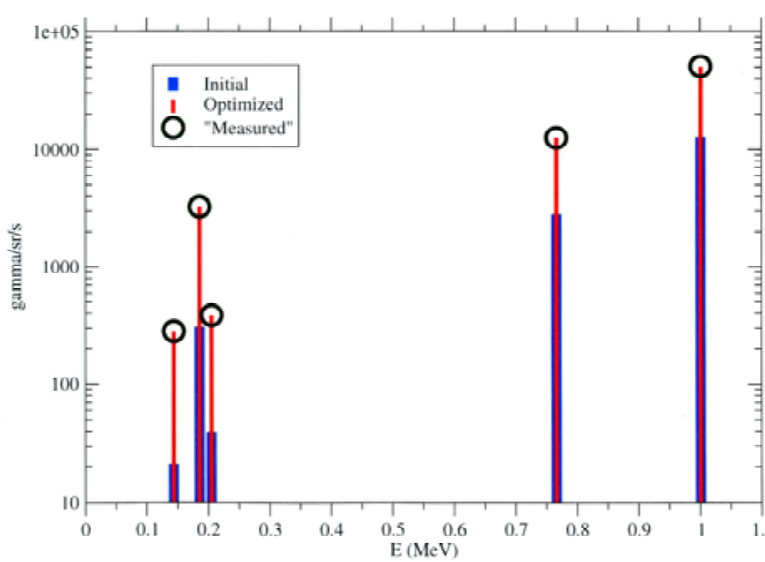

Fig. 7. Initial and optimized gamma line spectra compared to the measurement simulated by direct transport calculation.

\section{Conclusion}

Fast material thicknesses identification is performed using SGRD uncollided ray-tracing transport solver embedded in a gradient free stochastically restarted Powell iterative optimization loop.

We have presented the application of this methodology to a specific test problem. The code has been verified to work well using synthetic and measured data.

Future works will include the sensitivity analysis to measurement uncertainties, the use of other optimization parameters such as the source isotopic composition and the comparison with other optimization methods

\section{References}

1. J. A. Favorite, "Using the Schwinger Variational Functional for Solution of Inverse Transport Problems," Nucl. Sci. Eng., 146, 51-70 (2004)

2. K.C. Bledsoe, J.A. Favorite, and T. Aldemir, "Using the Levenberg-Marquardt Method for Solutions of Inverse Transport Problems in One- and TwoDimensional Geometries," Nucl. Tech., vol. 176, pp.106-126 (2011)
3. K.C. Bledsoe, J.A. Favorite, T. Aldemir, "Application of the Differential Evolution Method to Solving Inverse Transport Problems," Nucl. Sci. Eng., 169, pp. 208-221 (2011)

4. J.C. Armstrong, J.A. Favorite, "Identification of Unknown Interface Locations in Source/Shield System Using the Mesh Adaptive Direct Search Method," Trans. of the Am. Nucl. Soc., 106, pp. 375377 (2012)

5. J. Mattingly, D. J. Mitchell, "A Framework for the Solution of Inverse Radiation Transport Problems," IEEE Trans. Nucl. Sc. 57 (6), 3734-3743 (2010)

6. Ph. Humbert, B. Méchitoua, "Fast Gamma Ray Leakage Spectra Simulation," Proc. M\&C 2009, Saratoga Springs, New York, May 3-7, 2009, American Nuclear Society (2009) (CD-ROM)

7. K.M. Case, F. de Hoffmann, G. Placzek, "Introduction to the Theory of Neutron Diffusion," Vol.1, Los Alamos Scientific Laboratory report, USAEC (1953)

8. W. H. Press et al., Numerical Recipes, Cambridge University Press, Chapter 10 (1994)

9. R. Brent, "Algorithms for Minimization without Derivatives," Englewood Cliffs, N.J.: Prentice Hall, Chapter 7 (1973)

10. Ph. Humbert, B. Méchitoua, "Fast Computation of Pulse Height Spectra Using SGRD Code," Proc. ICRS-13 \& RPSD-2016, Paris, France (2016) 